\title{
Annihilation of NMSSM neutralinos in the Sun and neutrino telescope limits
}

\author{
Sergei Demidov \\ Institute for Nuclear Research of Russian Academy of Sciences, \\ prospect 60-th October 7A, Moscow 117312, Russia. \\ E-mail: demidov@ms2.inr.ac.ru \\ Olga Suvorova \\ Institute for Nuclear Research of Russian Academy of Sciences, \\ prospect 60-th October 7A, Moscow 117312, Russia. \\ E-mail: suvorova@cpc.inr.ac.ru
}

\begin{abstract}
We investigate neutralino dark matter in the framework of NMSSM performing a scan over its parameter space and calculating neutralino capture and annihilation rates in the Sun. We discuss the prospects of searches for neutralino dark matter in neutrino experiments depending on neutralino content and its main annihilation channel. We recalculate the upper limits on neutralino-proton elastic cross sections directly from neutrino telescopes upper bounds on annihilation rates in the Sun. This procedure has advantages as compared with corresponding recalcalations from the limits on muon flux, namely, it is independent on details of the experiment and the recalculation coefficients are universal for any kind of WIMP dark matter models. We derive $90 \%$ c.l. upper limits on neutralino-proton cross sections from the results of the Baksan Underground Scintillator Telescope.
\end{abstract}

KEYWORDS: dark matter theory, neutrino detectors. 


\section{Contents}

1. Introduction 1

2. Theoretical frames of the model 2

3. Neutralino-nucleon SI and SD cross sections

4. Neutralino capture rate in the Sun 6

5. Limits on neutralino-nucleon cross sections 10

6. Summary and conclusions 13

\section{Introduction}

Totality of phenomena observed in neighbouring and far cosmos indicates an existence of unusual forms of matter and energy, which constitute up to $95 \%$ of the energy density of the Universe. Nowadays experiments aimed at measurements of relic anisotropy [1], gravitation lensing [2] and dynamics of galaxies and clasters [3] allow to determine quantitatively dark matter density with a high precision and thereby greatly developed early F.Zwicky hidden mass hypothesis in the Universe [4].

In more probable cosmological model $\Lambda C D M$ (lambda cold dark matter) the contribution of baryonic matter in total density is less than $5 \%$, while the amount of dark matter is about 5 times larger. Experimentally the abundance of dark matter $\Omega_{\chi}$ expressed in units of critical density is fixed in a very narrow range $0.1109<\Omega_{\chi} h^{2}<0.1177$ [5], where $\mathrm{h}$ is the Hubble parameter in $100(\mathrm{~km} / \mathrm{s}) / \mathrm{Mpc}$ units.

Basic question about nature of dark matter is still open. There are no candidates in the Standard Model (SM) of particle physics which could play a role of the collisionless dark matter in the $\Lambda C D M$ cosmology paradigm. Among the most popular extensions of the SM are supersymmetry models (SUSY) which rather naturally provide with a stable WIMP (weakly interacting massive particle [6]) — the lightest neutralino. If thermally produced in the early Universe the lightest neutralino undergoes self-annihilations and freezes out at some relic density $\Omega_{\chi}$ (for review see, for example, [7, 8]). 
According to the electroweak (EW) theory weak interactions are mediated by gauge bosons while in SUSY models new particles with mass of order of EW scale also appear in the intermediate states. Due to the large mass of the gauge bosons weak interactions have very small interaction radius and the magnitude of the cross section of weak processes is a few orders smaller than that of processes mediated by strong or electromagnetic forces. Correspondingly, annihilation cross section of WIMP dark matter

particles ensures $\Omega_{\chi}$ density at EW scale. In this scenario WIMP masses are assumed to be between a few $\mathrm{GeV}$ and hundreds of $\mathrm{TeV}$.

In light of claimed spectral peculiarities the latest results of dark matter searches both in direct detections (DAMA [9], CDMS II [10]) and in cosmic ray experiments of gamma, electron-positron and proton-antiproton measurements (WMAP [1], EGRET [12], FERMI [13], INTEGRAL [14, PAMELA [15], ATIC [16]) have been interpreted by many authors as an evidence of the existence of light dark matter. In particular, neutralino dark matter in supersymmetric models were recently discussed in this context in papers [17, 18].

In this note we pay attention to neutralinos in a mass range about $20-2000 \mathrm{GeV} / c^{2}$ in the framework of Next to Minimal Supersymmetric Standard Model (NMSSM). In particular we consider two samples of its parameter space: mSUGRA-motivated models and general models without unification of parameters. With application of NMSSMTools package [19, 20, 21] we perform a scan over parameter space of the model and calculate capture and annihilation rates for neutralinos which could be trapped by the Sun. We compare the prospects for NMSSM neutralino searches at neutrino telescopes with that of in direct detection experiments. We also discuss the NMSSM benchmark points [22] in context of direct searches and data from neutrino telescopes. Then we consider a procedure to derive the limits on neutralino-nucleon cross section from the limits on annihilation rate in spirit of work [23] and use the data of the Baksan Underground Scintillator Telescope to derive the new limits.

\section{Theoretical frames of the model}

In supersymmetric models the neutralino is a Majorana fermion. The interactions of such a WIMP with ordinary matter can be both spin-dependent (SD) and spinindependent (SI). Moreover the dynamics of the annihilation and scattering processes depends on SUSY composition of the lightest neutralino, e.g. either it is a purely gaugino, higgsino or their mixture. We consider $\mathbb{Z}_{3}$ symmetric NMSSM model and use NMSSMTools package [19, 20, 21] to explore its parameter space for further analysis. 
The relevant part of superpotential $W$ has the form

$$
W=\lambda \hat{S} \hat{H}_{u} \cdot \hat{H}_{d}+\frac{1}{3} \kappa \hat{S}^{3}
$$

where $\hat{S}$ is a superfield which is a singlet with respect to SM gauge group and $\hat{H}_{u}$ and $\hat{H}_{d}$ are two Higgs doublets, $\hat{H}_{u}=\left(\hat{H}_{u}^{+}, \hat{H}_{u}^{0}\right)^{T}, \hat{H}_{d}=\left(\hat{H}_{d}^{0}, \hat{H}_{d}^{-}\right)^{T}$. Here we denote superfields by letters with hat and their scalar components by letters without it.

Soft SUSY breaking terms for this model read

$$
\begin{aligned}
-\mathcal{L}_{\text {soft }} & =m_{H_{u}}^{2} H_{u}^{\dagger} H_{u}+m_{H_{d}}^{2} H_{d}^{\dagger} H_{d}+m_{S}^{2} S^{*} S \\
& +\left(\lambda A_{\lambda} H_{u} \cdot H_{d} S+\frac{1}{3} \kappa A_{\kappa} S^{3}+\text { h.c. }\right)+\frac{1}{2}\left[M_{1} \tilde{B} \tilde{B}+M_{2} \tilde{W}^{i} \tilde{W}^{i}+M_{3} \tilde{G}^{a} \tilde{G}^{a}\right] .
\end{aligned}
$$

Here $m_{H_{u}}, m_{H_{d}}$ and $m_{S}$ are soft scalar masses, $A_{\lambda}, A_{\kappa}$ are trilinear coupling constants and $M_{1,2,3}$ are soft gaugino masses. The Higgs sector of the model contains the following independent parameters: $\lambda, \kappa, A_{\kappa}, A_{\lambda}, \tan \beta=\left\langle H_{u}\right\rangle /\left\langle H_{d}\right\rangle$ and $\mu \equiv \lambda\langle S\rangle$.

In the NMSSM the lightest mass eigenstate of neutralino can be written as a linear combination

$$
\chi=Z_{01} \tilde{B}+Z_{02} \tilde{W}^{3}+Z_{03} \tilde{H}_{u}^{0}+Z_{04} \tilde{H}_{d}^{0}+Z_{05} \tilde{s}
$$

of bino $\tilde{B}$, wino $\tilde{W}^{3}$, two higgsinos $\tilde{H}_{u}^{0}$ and $\tilde{H}_{d}^{0}$ and also singlino $\tilde{s}$ which is fermion component of the singlet superfield. Here coefficients $Z_{0 i}$ can be chosen as real-valued.

Below we will discuss the prospects of dark matter searches for two classes of the NMSSM model to be referred as Set 1 and Set 2.

- Set 1. Here we take mSUGRA-like NMSSM with unification of soft SUSYbreaking parameters which are specified at GUT scale

$$
M_{1,2,3} \equiv M_{1 / 2}, \quad M_{\tilde{q}_{i}}=M_{H_{i}} \equiv m_{0}, \quad A_{i} \equiv A_{0},
$$

where $m_{\tilde{q}_{i}}$ are soft squarks masses and $A_{i}$ are all soft trilinear coupling constants except for $A_{\kappa}$. We take these parameters in the following ranges:

$0 \mathrm{GeV}<m_{0}<5000 \mathrm{GeV}, 0 \mathrm{GeV}<M_{1 / 2}<4000 \mathrm{GeV}$, $-4000 \mathrm{GeV}<A_{0}, A_{\kappa}<4000 \mathrm{GeV}$, sign $\mu>0,0.0<\lambda<0.7,1.7<\tan \beta<54$.

The dark matter in the mSUGRA-like NMSSM was already discussed in Refs. 24, 25. In this case $A_{\kappa}$ is considered as an independent parameter and $\kappa$ is obtained from minimization of the scalar potential (see Ref. [24] for details).

- Set 2. Here we assume general NMSSM without unification of the soft terms and adopt the following ranges for parameters specified at SUSY breaking scale: 
$0 \mathrm{GeV}<\mu, M_{1}, M_{2}<2000 \mathrm{GeV}$,

$300 \mathrm{GeV}<M_{3}<3000 \mathrm{GeV},-2000 \mathrm{GeV}<A_{\lambda}, A_{\kappa}<2000 \mathrm{GeV}$,

$0.0<\lambda<0.7,0.0<\kappa<0.6,1.7<\tan \beta<54$; soft sfermion masses and sfermion trilinear terms are varied within $\pm 3 \mathrm{TeV}$. The ranges of defined parameters allow to construct rather light neutralinos. In a view of the prospects for the indirect dark matter searches there was related discussion in Ref. [26] for $m_{\chi}<100 \mathrm{GeV} / c^{2}$, while we do not restrict ourselves by only light neutralinos.

We take $m_{t}=171.4 \mathrm{GeV}$ for the mass of top-quark. For both model classes we scan over their parameter space by use NMSSMTools [19, 20, 21]. All unphysical or phenomenologically unacceptable models are rejected by checks for absence of Landau pole up to GUT scale for $\lambda$ and $\kappa$, as well for absence of unphysical global minimum of the scalar potential in Higgs sector and also for absence of colour breaking minima. Finally, we follow the NMSSMTools implications of the experimental constraints (full list of them can be found in Refs. [19, 20, 21]). Note, however, that we do not impose the condition that the SUSY contribution to the anomalous magnetic moment of muon should explain the present $3 \sigma$ difference between SM prediction and BNL results. For our further analysis we take the lightest neutralino as potential dark matter candidates if their relic abundance is $\Omega_{D M}<0.3$ and finally, we obtain about $5 \cdot 10^{5}$ phenomenologically accepted models for each set of parameters presented above.

\section{Neutralino-nucleon SI and SD cross sections}

Among relevant physical quantities spin-dependent and spin-independent neutralinonucleon scattering cross sections are more probable to be tested in experimental searches

for dark matter interactions. The expected signal rate has direct dependence on neutralino scattering cross sections off ordinary nucleons not only in direct detection experiments but as well as in neutrino telescopes. In the case of annihilations of neutralino pairs inside the Sun where solar chemical composition contains more than $73 \%$ of hydrogen [27, either SD or SI parts of the cross section could give dominant contribution to neutralino capture and annihilation rates.

The effective interaction lagrangian responsible for the spin-dependent part of the neutralino-nuclei cross section is

$$
\mathcal{L}_{A}=d_{q}\left(\bar{\chi} \gamma^{\mu} \gamma^{5} \chi\right)\left(\bar{q} \gamma_{\mu} \gamma^{5} q\right)
$$

and the expression for SD cross section has the form

$$
\sigma_{S D}=\frac{32}{\pi} G_{F}^{2} m_{r}^{2} \Lambda^{2} J(J+1)
$$


where $m_{r}$ is the reduced neutralino mass $m_{r}=\frac{m_{\chi} m}{m_{\chi}+m}, m$ and $J$ are the mass and the spin of the nucleus respectively and

$$
\Lambda=\frac{1}{J}\left(a_{p}\left\langle S_{p}\right\rangle+a_{n}\left\langle S_{n}\right\rangle\right)
$$

where $\left\langle S_{p}\right\rangle$ and $\left\langle S_{n}\right\rangle$ are the spin content of the proton and neutron while

$$
a_{p}=\sum_{q} \frac{d_{q}}{\sqrt{2} G_{F}} \Delta_{q}^{(p)}, \quad a_{n}=\sum_{q} \frac{d_{q}}{\sqrt{2} G_{F}} \Delta_{q}^{(n)} .
$$

As default values for the deltas we use

$$
\Delta_{u}^{(p)}=0.842, \quad \Delta_{d}^{(p)}=-0.427, \quad \Delta_{s}^{(p)}=-0.085
$$

The axial-vector coefficients for neutron can be obtained from the values (3.5) by corresponding isospin rotation.

The spin-independent interactions of neutralino with nucleus are generated by the following effective interaction lagrangian

$$
\mathcal{L}=c_{q}(\bar{\chi} \chi)(\bar{q} q)
$$

We do not discuss here the dependence of physical quantities on parameters of the NMSSM lagrangian and so we do not present explicit expressions for $d_{q}$ and $c_{q}$. The details of the calculation of these coupling constants from NMSSM lagrangian can be found e.g. in Ref. 28.

The SI cross section of neutralino on nucleus can be written as

$$
\sigma_{\mathrm{SI}}=\frac{4 m_{r}^{2}}{\pi}\left(Z f_{p}+(A-Z) f_{n}\right)^{2}
$$

where $\mathrm{Z}$ and $\mathrm{A}$ are the charge and the atomic number of the nuclei, respectively, and the nucleon formfactors have the form

$$
\frac{f_{N}}{m_{N}}=\sum_{q=u, d, s} f_{T_{q}}^{(N)} \frac{c_{q}}{m_{q}}+\frac{2}{27} f_{T G}^{(N)} \sum_{q=c, b, t} \frac{c_{q}}{m_{q}}, \text { where } f_{T G}^{(N)}=1-\sum_{q=u, d, s} f_{T_{q}}^{(N)} .
$$

The parameters $f_{T_{q}}^{(N)}$ are defined from expressions

$$
m_{N} f_{T_{q}}^{(N)}=\left\langle N\left|m_{q} \bar{q} q\right| N\right\rangle=m_{q} B_{q}^{(N)}
$$

As discussed in several recent papers [34, 35] the main uncertainty in determination of SI cross section comes from the pion-nucleon scattering sigma term, $\sigma_{\pi N}$, which 
determines the coefficients $f_{T_{q}}$ as follows

$$
\begin{aligned}
f_{T_{u}} & =\frac{2 \sigma_{\pi N}}{m_{N}\left(1+\frac{m_{d}}{m_{u}}\right)\left(1+\frac{B_{d}}{B_{u}}\right)}, \quad f_{T_{d}}=\frac{2 \sigma_{\pi N}}{m_{N}\left(1+\frac{m_{u}}{m_{d}}\right)\left(1+\frac{B_{u}}{B_{d}}\right)}, \\
f_{T_{s}} & =\frac{\left(\frac{m_{s}}{m_{d}}\right) \sigma_{\pi N} y}{m_{N}\left(1+\frac{m_{u}}{m_{s}}\right)}, y \equiv \frac{2 B_{s}}{B_{u}+B_{d}} .
\end{aligned}
$$

We use $m_{u} / m_{d}=0.553$ and $m_{s} / m_{d}=18.9$. Following Ref. [28] we define the quantity

$$
z=\frac{B_{u}-B_{s}}{B_{d}-B_{s}} \approx 1.49
$$

and strange quark density

$$
y=1-\frac{\sigma_{0}}{\sigma_{\pi N}}
$$

where

$$
\sigma_{0}=\frac{m_{u}+m_{d}}{2}\left(B_{u}+B_{d}-2 B_{s}\right)
$$

and $\sigma_{\pi N}$-term can be written as

$$
\sigma_{\pi N}=\frac{m_{u}+m_{d}}{2}\left(B_{u}+B_{d}\right)
$$

The recent estimates show that the value of $\sigma_{\pi N}$ lies in the interval $55 \mathrm{MeV}<\sigma_{\pi N}<$ $73 \mathrm{MeV}$ and also it is suggested that $\sigma_{0}=35 \pm 5 \mathrm{MeV}$ 29. We use $\sigma_{\pi N}=64 \mathrm{MeV}$ as default value for our calculations. We will back to the discussion of influence of known uncertainties in the nuclear matrix elements on the neutralino annihilation rates in the next sections.

\section{Neutralino capture rate in the Sun}

At present the estimated local density of relic dark matter in the solar system $\rho_{\chi}^{\text {loc }}$ is about $0.3 \mathrm{GeV} / \mathrm{cm}^{3}$. The speeds of halo WIMPs crossing the ecliptic plane are nonrelativistic with mean velocity $\bar{v} \sim 270 \mathrm{~km} / \mathrm{s}$ (the root-mean-square of the dispersion) in the Maxwell-Boltzmann distribution. As far as they are gravitationally trapped by the Sun, a multiple scattering of WIMP off solar matter is likely happened with energy losses. Due to these interactions WIMPs can be captured inside the Sun. Their orbital motion changes to smaller radius and toward the dense centre, where they 
finally settle and accumulate and as well self-annihilate at a distance smaller than annihilation length. During the lifetime of the Sun these two processes of capture and annihilation may reach approximate equilibrium and in this case accumulated number of WIMP does not change in time. One of the prominent signature of the neutralino annihilations is high energy neutrinos generated in decays of annihilation products, since these neutrino events can be detected by neutrino telescopes.

Such a scheme has been considered by A.Gould [30] and, e.g., later in detailed paper [31], see also references therein. Follow them we can obtain neutralino annihilation rate from the capture rate in the Sun by solving known evolution equation for the number of WIMPs

$$
\frac{d N}{d t}=C_{C}-C_{A} N^{2}
$$

where $C_{C}$ is the capture rate of WIMP and $C_{A}$ is the specific annihilation rate related to the Sun volume. The second term in equation (4.1) has the meaning of the rate of WIMPs pair annihilations: $2 \cdot \Gamma_{A} \equiv C_{A} N^{2}$. The process of WIMPs evaporation does not included in (4.1) since it is negligible for WIMP mass larger a few $\mathrm{GeV} / c^{2}$ [30, 32]

It is easy to show that when equilibrium time $t_{e q}=\left(C_{C} \cdot C_{A}\right)^{-1 / 2}$ is much less than the live time of the solar system the annihilation rate is the half of capture rate

$$
\Gamma_{A}=\frac{C_{C}}{2} \cdot \tanh ^{2}\left(t_{\mathrm{Sun}} / t_{\mathrm{eq}}\right)
$$

since the number of WIMPs is solved as

$$
N=\sqrt{\frac{C_{\mathrm{C}}}{C_{\mathrm{A}}}} \cdot \tanh \left(t_{\mathrm{Sun}} / t_{\mathrm{eq}}\right) .
$$

The quantity $C_{\mathrm{A}}$ is determined by following expression [30, 32

$$
C_{\mathrm{A}}=\frac{\left\langle\sigma_{\mathrm{ann}} \cdot v\right\rangle}{V_{\mathrm{Sun}}} \cdot\left(\frac{m_{\chi}}{100 \mathrm{GeV}}\right)^{3 / 2},
$$

where $\left\langle\sigma_{a n n} \cdot v\right\rangle$ is the neutralino total annihilation cross section thermally averaged with the relative neutralino velocity $v$ and $V_{\text {Sun }}=5.7 \cdot 10^{27} \mathrm{~cm}^{3}$ is the effective volume of the Sun.

For calculation of the capture rate we use simplified model [33] of the solar potential which can be parametrized by escape velocity $v_{e s}(r)$ on distance $r$ from the center of the Sun as follows

$$
v_{e s}^{2}(r)=v_{c}^{2}-\frac{M(r)}{M_{\odot}}\left(v_{c}^{2}-v_{s}^{2}\right),
$$


where $v_{c}=1354 \mathrm{~km} / \mathrm{s}, v_{s}=795 \mathrm{~km} / \mathrm{s}$ and $M(r)$ is the mass of the Sun inside a sphere of radius $r$. In this approximation the capture rate can be written in an analytical form [30, 33, 7]

$$
\begin{aligned}
C= & \frac{M_{\odot} \rho_{\chi} \bar{v}}{4 \sqrt{6} m_{\chi}^{2} \eta} \sum_{i} f_{i} \sigma_{i} \frac{\left(m_{\chi}+m_{i}\right)^{2}}{m_{i}^{2} a}\left\{\frac{2 \exp \left(-a \hat{\eta}^{2}\right)}{(1+a)^{1 / 2}} \operatorname{erf}(\hat{\eta})-\frac{\exp \left(-a \hat{\eta}^{2}\right)}{(1+a)^{3 / 2}\left(A_{c}^{2}-A_{s}^{2}\right)} \times\right. \\
& \times\left\{\left(\hat{A}_{+} \hat{A}_{-}-\frac{1}{2}-\frac{1+a}{a-b}\right)\left(\operatorname{erf}\left(\hat{A}_{+}\right)-\operatorname{erf}\left(\hat{A}_{-}\right)\right)+\frac{1}{\sqrt{\pi}}\left(\hat{A}_{-} \mathrm{e}^{-\hat{A}_{+}}-\hat{A}_{+} \mathrm{e}^{-\hat{A}_{-}}\right)\right\}_{A_{s}}^{A_{c}} \\
& \left.+\frac{\exp \left(-b \check{\eta}^{2}\right)}{(a-b)(1+b)^{1 / 2}\left(A_{c}^{2}-A_{s}^{2}\right)}\left\{\mathrm{e}^{-(a-b) A^{2}}\left[2 \operatorname{erf}(\check{\eta})-\operatorname{erf}\left(\check{A}_{+}\right)+\operatorname{erf}\left(\check{A}_{-}\right)\right]\right\}_{A_{s}}^{A_{c}}\right\}
\end{aligned}
$$

where $m_{i}$ is the mass of $i$-th chemical element, $f_{i}$ is its mass content in the Sun, $\sigma_{i}$ is total (the sum of SI and SD contributions) neutralino-nuclei elastic cross section, $A(r)=\frac{3 v_{e s}^{2}(r)}{2 \bar{v}^{2}} \frac{\mu}{\mu_{-}}, A_{s}=A_{\mid v_{e s}=v_{s}}$ and $A_{c}=A_{\mid v_{e s}=v_{c}}$ and the following notations are introduced

$$
\begin{array}{r}
\hat{A}_{ \pm}=\hat{A} \pm \hat{\eta}, \quad \check{A}_{ \pm}=\check{A} \pm \check{\eta}, \quad \hat{A}=A(1+a)^{1 / 2}, \quad \check{A}=A(1+b)^{1 / 2}, \quad a=\frac{m_{\chi} \bar{v}^{2}}{3 E_{0}} \\
\hat{\eta}=\frac{\eta}{(1+a)^{1 / 2}}, \quad \check{\eta}=\frac{\eta}{(1+b)^{1 / 2}}, \quad b=\frac{\mu}{\mu_{+}} a, \quad \mu=\frac{m_{\chi}}{m_{i}}, \quad \mu_{+}=\frac{\mu+1}{2}
\end{array}
$$

where $E_{0}=\frac{3 \hbar^{2}}{2 m_{N} R^{2}}$ and $R=\left[0.91\left(\frac{m_{i}}{\mathrm{GeV}}\right)^{1 / 3}+0.3\right] \times 10^{-13} \mathrm{~cm}$. Here we assume the isothermal spherical model for halo WIMPs distribution, where $\eta$ is equal to 1 . Expression (4.6) is obtained with additional assumption the all chemical elements are distributed uniformly in the Sun. This allows to perform analytic integration over inner part of the Sun and results in the lengthy term in outer curly brackets. In our calculations we use uniformly distributed solar elements obtained from GS98 solar model presented in [27.

Eq. (4.6) shows explicitly the direct functional dependence of the capture rate on using approximations and values of scattering cross sections, velocity distribution, local dark matter density and solar model. In discussion of physical implications of the calculated capture rate (and, therefore, annihilation rate) one should bear in mind all related uncertainties. In particularly, according to recent analysis in Ref. [35], error in determination of $\Delta_{s}^{(p)}$ in calculation of SD cross section results in $10 \%$ uncertanty in annihilation rate, while lack of exact knowledge of $\sigma_{\pi N}$ can give rather substantial errors (up to order of magnitude) in annihilation rate if capture rate is saturated by SI interactions. The accuracy of using the approximation (4.5) and assumption about uniformly distributed elements in the Sun with respect to e.g. AGSS09 solar profile [27] is within $11 \%$. Also as it follows from cosmological N-body simulations [36] the 


\begin{tabular}{|l|c|c|c|c|}
\hline Point & P1,P2 & P3 & P4 & P5 \\
\hline$m_{\text {WIMP }}, \mathrm{GeV} / \mathrm{c}^{2}$ & 208.2 & 208.3 & 90.7 & 70.5 \\
\hline$\Gamma_{A}, \mathrm{~s}^{-1}$ & $3.8 \cdot 10^{17}$ & $2.4 \cdot 10^{18}$ & $1.8 \cdot 10^{23}$ & $1.6 \cdot 10^{22}$ \\
\hline annihilation channel & $t \bar{t}$ & $t \bar{t}$ & $W^{+} W^{-}$ & $b \bar{b}$ \\
\hline$\sigma_{S I, p}, \mathrm{pb}$ & $2.4 \cdot 10^{-10}$ & $6.7 \cdot 10^{-10}$ & $6.6 \cdot 10^{-7}$ & $1.3 \cdot 10^{-7}$ \\
\hline$\sigma_{S D, p}, \mathrm{pb}$ & $3.4 \cdot 10^{-9}$ & $7.4 \cdot 10^{-9}$ & $8.5 \cdot 10^{-4}$ & $2.6 \cdot 10^{-5}$ \\
\hline \hline
\end{tabular}

Table 1: Benchmark NMSSM points.

local dark matter density $\rho_{\chi}^{\text {loc }}$ might be about $20 \%$ larger than usually adopted value $0.3 \mathrm{GeV} / \mathrm{cm}^{3}$.

Now we consider the NMSSM benchmark points proposed and discussed in the paper 22] in the search strategies at the LHC. In the Table 11 we present the physical quantities relevant for the dark matter searches in these models: the annihilation rates, the main annihilation channel and also SI and SD elastic cross sections. We note in passing that the models P1-P3 and P5 correspond to almost pure bino dark matter, while in the models P5 the lightest neutralino is singlino. Comparison with the latest results of the direct searches (see Sec. 5) shows that even with account of the error bars (see next section for an illustrative figure) the points P4 and P5 are already excluded, while models P1-P3 can not be probed in the nearest future direct searches and at neutrino experiments.

Annihilation rates $\Gamma_{A}$ calculated in described frames of Set 1 and Set 2 models are plotted in Fig.1 as a function of neutralino mass. The upper limits $\Gamma_{A}^{\text {UppLim }}$ obtained at $90 \%$ c.l. in neutrino experiments of the Baksan [37] and the AMANDA [38] collaborations are also shown. In Fig. 1 w we markup points of the NMSSM models by colours correspondent to different dominant annihilation channels, with more than 0.5 branching ratio in each case. There is obvious difference between these two SUSY samples from point of view annihilation branches. Annihilations to $b \bar{b}$ in mSUGRA-like model overcomes others for almost all neutralino masses. Consequently, these $b \bar{b}$-like models can be excluded only by low energy threshold experiments as the Baksan [37, 39, 40] and the Super Kamiokande [41] telescopes. A role of $\tau^{+} \tau^{-}$channel is not remarkable in mSUGRA-like model for low neutralino masses, while we find expected competition of branching ratios of $b \bar{b}$ and $\tau^{+} \tau^{-}$for Set 2. In mass range near $60-80 \mathrm{GeV} / c^{2}$ there is a decrease in the number of points due to closeness of possible value of the scalar Higgs mass to double neutralino mass. However the models inside this mass interval have a high potential to be seen or excluded by neutrino telescopes. The same conclusion can be drawn for scalars channels (i.e., annihilation of neutralinos to scalar and pseudoscalar Higgs bosons) for Set 2 .

In the frames of considered models the lightest neutralino could be singlino with 
light mass even less than $50 \mathrm{GeV} / c^{2}$. Not many singlinos satisfy condition $\Omega_{\chi}<0.3$ and only small amount of them survive imposing $3 \sigma$ WMAP density cut as it can be seen in Fig. 2. However singlinos appear to be among the most promising candidates in Set 2 in searching for neutralino annihilation signature in neutrino telescopes. To see which annihilation channel plays dominant role we show the rest of the models in Fig. 3 separately and respectively to colours in Fig. 11.

We note that for neutralino mass less than $80 \mathrm{GeV} / c^{2}$ main annihilation channels are quark and lepton pairs. Neutralino annihilating mainly to scalars has larger annihilation rate then one with leptonic channel. As we see the Baksan exclusion line of $\Gamma_{A}^{\text {UppLim }}$ goes yet closely to model candidates. The AMANDA lower (solid) line of limit could be compared here only with $\tau^{+} \tau^{-}, W^{+} W^{-}$and $t \bar{t}$ points while for $b \bar{b}$ channels (green points) their $\Gamma_{A}^{U p p L i m}$ limits [38] (dashed line) are in two orders higher.

\section{Limits on neutralino-nucleon cross sections}

The basic conclusion followed from the equation (4.1) is that annihilation rate $\Gamma_{A}$ is determined by WIMPs scattering cross sections off solar matter and in equilibrium does not depend on their annihilation cross sections

$$
\Gamma_{A}=\frac{C_{C}}{2}
$$

In this case annihilation rate (4.2) can be divided into two pieces which correspond to different type of contribution (SI or SD) to neutralino-nuclei interactions

$$
\Gamma_{A}=\Gamma_{A}\left(\sigma_{S I}\right)+\Gamma_{A}\left(\sigma_{S D}\right)
$$

where $\Gamma_{A}\left(\sigma_{S I}\right)$ and $\Gamma_{A}\left(\sigma_{S D}\right)$ are determined by (5.1) and (4.6), where one should keep only SI or SD part of neutralino-nuclei cross section respectively. To demonstrate the relative importance of SI and SD contributions we plot their ratio in Fig. 14 for Set 1 and Set 2 in dependence on neutralino mass and dominant annihilation channels (here and below we present in figures only the models within $3 \sigma$ WMAP region for dark matter abundance). As expected the axial interactions play dominant role for relatively light neutralinos $\left(m_{\chi} \lesssim 200-500 \mathrm{GeV}\right)$ while annihilation rate for heavier neutralino is saturated in main part by scalar interactions. Hence for large neutralino masses the uncertainties in the calculations of the annihilation rate increase.

As it has been shown by G. Wikstrom and J. Edsjo [23], if the WIMP's processes in the Sun reach exact equilibrium one can derive upper limits on WIMP scattering cross sections from upper limits on upward going muon fluxes obtained by neutrino 
telescopes. Consequently these results simplify comparison with direct WIMP searches via nuclear recoil reactions.

Early in the Baksan neutralino search [40] it was mentioned a preference of limits on annihilation rate $\Gamma_{A}$ for the purpose of comparison with model prediction because muon flux $\Phi_{\mu}$ calculated for some model depends on details of the experiment, e.g. on its energy threshold. The magnitude of annihilation rate gives the scale for neutrino flux from a distant source (like the Sun at the distance R), that is

$$
\frac{d N_{\nu_{j}}}{d E_{\nu}}=\frac{\Gamma_{A}}{4 \pi R^{2}} \sum_{i} B_{i} \frac{d N_{\nu_{j}}^{i}}{d E_{\nu}}, \quad \nu_{j}=\nu_{\mu}, \bar{\nu}_{\mu}
$$

where $d N_{\nu_{j}}^{i} / d E_{\nu}$ is the differential spectrum of $\mathrm{j}$-th neutrinos at the surface of the the Sun produced in i-th annihilation channel. The branching ratios $B_{i}$ depend on SUSY parameters as we show above and obviously drive the shape of the final neutrino spectrum, which could be either mainly "soft"-like from annihilation channels into two quarks (e.g. a pair of $b \bar{b}$ ) or mainly "hard"-like from lepton decay channels of two bosons (e.g. a pair of $W^{+} W^{-}$).

Definition of an upper limit on neutralino annihilation rate is read from the equation 40

$$
\Gamma_{A}^{U p p L i m}=\frac{N^{U p p L i m}(\vartheta)}{T \cdot \varepsilon_{\vartheta}} \times \frac{1}{P_{a n n}},
$$

where $N^{U p p \operatorname{Lim}}(\vartheta)$ is an experimental limit at a given confidence level on number of events and for a given half-angle of open cone $\vartheta$ toward the Sun, T is a time of observation, and $\varepsilon_{\vartheta}$ is a fraction of total number of neutrino events collected within the angle $\vartheta$ from expected neutrino source. A value $P_{a n n}$ is neutrino telescope probability to detect muon per one pair of neutralino annihilation. Here the upward going muon is produced in charged current neutrino interactions in the Earth. To get the probability $P_{a n n}$ the detailed Monte Carlo simulations are required for the detector response with generator of neutrino energy spectrum (5.3) and further propagation of oscillating and interacting neutrinos in matter (see e.g. Refs. 49]) and also induced muons on the route to the detector. From these studies a relation between neutralino mass and the angle $\vartheta$ could be found. The latest results with $90 \%$ c.l. upper limits of annihilation rates in the Sun are presented by both the AMANDA [38] and the IceCube 42 collaborations and as well by the Baksan Underground Scintillator Telescope in Ref. [37.

We suggest to implement obtained upper limits on annihilation rate $\Gamma_{A}^{\text {UppLim }}$ to put upper limits on neutralino SD and SI scattering cross sections off protons (see also Eq.(15) in Ref. [23])

$$
\sigma_{S D, p}^{U p p \operatorname{Lim}}\left(m_{\chi}\right)=\lambda^{S D}\left(m_{\chi}\right) \cdot \Gamma_{A}^{U p p L i m}\left(m_{\chi}\right)
$$




\begin{tabular}{|c|c|c|c|c|}
\hline$m_{\text {WIMP }}, \mathrm{GeV} / c^{2}$ & $\lambda^{S I}, \mathrm{pb} / \mathrm{s}^{-1}$ & $\lambda^{S D}, \mathrm{pb} / \mathrm{s}^{-1}$ & $\sigma_{S I, p}^{\text {Uppim }}, \mathrm{pb}$ & $\sigma_{S D, p}^{\text {Up Lim }}, \mathrm{pb}$ \\
\hline 12.6 & $4.24 \cdot 10^{-30}$ & $2.53 \cdot 10^{-28}$ & $6.79 \cdot 10^{-3}$ & 0.405 \\
\hline 32.6 & $6.95 \cdot 10^{-30}$ & $9.96 \cdot 10^{-28}$ & $9.73 \cdot 10^{-4}$ & 0.139 \\
\hline 82.7 & $1.66 \cdot 10^{-29}$ & $5.05 \cdot 10^{-27}$ & $5.81 \cdot 10^{-5}$ & 0.0177 \\
\hline 210.3 & $5.22 \cdot 10^{-29}$ & $3.00 \cdot 10^{-26}$ & $5.74 \cdot 10^{-7}$ & $3.3 \cdot 10^{-4}$ \\
\hline 533.9 & $2.13 \cdot 10^{-28}$ & $1.87 \cdot 10^{-25}$ & $5.33 \cdot 10^{-7}$ & $4.68 \cdot 10^{-4}$ \\
\hline 1356.7 & $1.11 \cdot 10^{-27}$ & $1.19 \cdot 10^{-24}$ & $1.28 \cdot 10^{-6}$ & $1.37 \cdot 10^{-3}$ \\
\hline 3462.8 & $6.64 \cdot 10^{-27}$ & $7.74 \cdot 10^{-24}$ & $3.85 \cdot 10^{-6}$ & $4.49 \cdot 10^{-3}$ \\
\hline \hline
\end{tabular}

Table 2: Coefficients $\lambda^{S D}, \lambda^{S I}$ and the upper limits $\sigma_{S D, p}^{U p p L i m}, \sigma_{S I, p}^{U p p L i m}$ at $90 \%$ c.l. on elastic $\chi$-proton cross sections recalculated from the Baksan $90 \%$ c.l. upper limits on annihilation rates.

$$
\sigma_{S I, p}^{U p p \operatorname{Lim}}\left(m_{\chi}\right)=\lambda^{S I}\left(m_{\chi}\right) \cdot \Gamma_{A}^{U p p \operatorname{Lim}}\left(m_{\chi}\right) .
$$

Coefficients for these calculations are following

$$
\lambda^{S D}\left(m_{\chi}\right)=\frac{\sigma_{S D, p}^{\chi}}{\Gamma_{A}^{\chi}\left(\sigma_{S D}\right)}, \quad \lambda^{S I}\left(m_{\chi}\right)=\frac{\sigma_{S I, p}^{\chi}}{\Gamma_{A}^{\chi}\left(\sigma_{S I}\right)} .
$$

They can be obtained from formulae above with the assumption of dominance either spin-dependent or spin-independent interactions. Clearly, these coefficients in contrast with ratios $\Phi_{\mu} / \Gamma_{A}, \sigma_{S I, p} / \Phi_{\mu}$ and $\sigma_{S D, p} / \Phi_{\mu}$ presented in paper [23], are universal in the sense that they do not depend on the experimental setup (e.g., on energy threshold) and on the model of the WIMP dark matter, although it still contains uncertainties of astrophysical values (see Eq. (4.6)). Coefficients $\lambda^{S D}$ and $\lambda^{S I}$ as functions of neutralino mass $m_{\chi}$ are plotted in Fig.5.

In Table 2 we present the upper limits on neutralino-proton elastic cross sections $\sigma_{S D, p}^{U p p L i m}$ and $\sigma_{S I, p}^{\text {Uppim }}$ which we derive from the Baksan $90 \%$ c.l. upper limits on annihilation rate [37] by use coefficients (5.7) which are also shown in Table 2. The neutralino mass values are the same as in the Baksan final table 40].

Graphical view of the obtained limits on cross sections is shown in Fig. 6. Here for comparison (and as well to check our procedure) we show both the upper limits on SI and SD cross sections presented by the AMANDA experiment in [38] and our current recalculations from the AMANDA upper limits on annihilation rates [38. We observe nice coincidence. Therefore coefficients $\lambda^{S D}$ and $\lambda^{S I}$ presented in Table 2 and in Fig. could be used in recalculation of annihilation rate limits for other neutrino telescopes (such as the Baikal NT-200 [46] and ANTARES [47]) in wide range of neutralino masses.

In Figs. 7 and 8 neutralino-proton SI and SD cross sections calculated for NMSSM models are shown with the strongest experimental upper limits $\sigma_{S I, p}^{\text {UppLim }}$ and $\sigma_{S D, p}^{\text {Upp Lim }}$ obtained at $90 \%$ c.l. in direct detections (CDMS [10], XENON10 [43], ZEPLIN-III [44] 
and PICASSO [45]) and neutrino telescopes (AMANDA [38], Baksan [37 and IceCube [42]). We implement the DMTools 48 database to plot listed upper limits. Although main part of the DAMA [9] allowed ranges are excluded by different direct experiments, these $3 \sigma$ regions with and without ion channeling are also shown as shadowed. One can see that the SI limits from the neutrino detectors are able to close the DAMA results for neutralino mass around $80-100 \mathrm{GeV} / c^{2}$. The Baksan upper limits on SD cross sections close the values covered by the DAMA for neutralino masses down to $12 \mathrm{GeV} / c^{2}$. At the lightest mass the Baksan $\sigma_{S D, p}^{\text {Uppim }}$ level is the same as the latest results of the ZEPLIN-III 44 and PICASSO 45. Finally the Baksan limits on SD cross sections resolve the "gap" 23] between limits of the direct detections and predicted SUSY models, here the NMSSM models.

In Figs. 7 and 8 we markup the different neutralino type by colours. Namely we identify the lightest neutralino as bino if $Z_{01}^{2}>0.5$, as wino if $Z_{02}^{2}>0.5$, as higgsino if $Z_{03}^{2}+Z_{04}^{2}>0.5$ and as singlino if $Z_{05}^{2}>0.5$. For mSUGRA-type models (Set 1) the lightest neutralino is bino for almost entire parameter space. However for the Set 2 there are quite a lot of models in which dark matter can be wino, higgsino or singlino. Note that in Fig. 8 we drop all models which are excluded by the limits on SI neutralino-proton cross section shown in Figs. 7 with account of $\sigma_{\pi N}$ uncertainty. Namely we plot only those points which are not excluded at least for some values of $\sigma_{\pi N}$ from the interval 55-73 MeV. Also one can see that population of bino-like neutralinos in the NMSSM has better prospects to be found or excluded in experimental search by both direct methods and neutrino detections. Two benchmark points P4 and P5 are shown with error bars for SI cross sections. From SI limits in Figs. 7 one can conclude about exclusion of $\mathrm{P} 4$ and $\mathrm{P} 5$ models by direct detection experiments. Also the point P4 in Figs. 8 is close to being excluded by SD limits from the neutrino telescopes.

\section{Summary and conclusions}

We discussed here the prospects of neutrino telescope searches for neutralino dark matter in the framework of the NMSSM. In our study we analyse two types of the NMSSM scenarios, namely, mSUGRA-like models and general NMSSM without unification of the soft terms and perform a scan over the NMSSM parameter space with using NMSSMTools package. We have found that the dominant annihilation channels in the mSUGRA-like models are very different from those of the general NMSSM. In case of the mSUGRA models the lightest neutralino is mainly bino and it annihilates dominantly into $b \bar{b}$ pairs. However, neutralino annihilating into $W^{+} W^{-}$and $t \bar{t}$ pairs is also very promising for searches at neutrino telescopes. For general NMSSM model we have found expected competition between $b \bar{b}$ and $\tau^{+} \tau^{-}$channels of annihilation for 
neutralino mass less than mass of $\mathrm{W}$-boson, while for heavier neutralino $W^{+} W^{-}$and $t \bar{t}$ channels are comparable with those into scalar pairs. We found that NMSSM neutralinos with mass less about $200 \mathrm{GeV} / c^{2}$ scatter off nucleons in the Sun mainly by axial interactions and with increasing of the mass value the part of the scalar interactions also increases such that at masses higher than $500 \mathrm{GeV} / \mathrm{c}^{2}$ it is almost spin-independent scattering. In general, for both types of NMSSM models singlino and bino are the most probable models to be tested either at the neutrino telescopes with low energy threshold through neutralino axial-vector (SD) interactions in the Sun or in the direct detection experiments sensible to scalar (SI) neutralino interactions.

Depending on neutralino properties there are essentially different prospects in search for them at neutrino telescopes with low and high energy thresholds. The type of annihilation products determines fractions of low (soft) and high (hard) energy spectra of outcoming particles including neutrinos. Probability of neutrino telescope to detect neutrinos from predicted neutralino models absorbs dependence on energy threshold of the detector for neutrino induced upward going muons. Presently among the operating neutrino telescopes the Baksan Underground Scintillator Telescope and the Super Kamiokande have low energy thresholds (about $1 \mathrm{GeV}$ ). The Baksan 90\% c.l. upper limits on annihilation rate in the Sun result from absence neutralino signal in all expected mass range from dozen $\mathrm{GeV} / c^{2}$. The large detectors with high thresholds e.g. AMANDA or IceCube present the upper limits on annihilation rates for separated annihilation branches with soft and hard neutrino spectra. Therefore all limits can be compared only in context of a particular neutralino annihilation channel.

Finally, we have discussed a relation between the limits on neutralino annihilation rate in the Sun and the limits on neutralino-proton SI and SD cross section. Under assumption of equilibrium of the neutralino processes in the Sun we derive 90\% c.l. upper limits on SI and SD neutralino-proton cross sections from the upper limits on annihilation rates for the Baksan Underground Scintillator Telescope. Also we tabulate the relevant coefficients for calculation the above limits, since they are independent on the details of a particular experiment and the type of WIMP dark matter model.

\section{Acknowledgments}

We thank S.P. Mikheev for useful remarks and discussions. The work was supported in part by the Russian Found for Basic Research Grant 09-02-00163a. The work of S.D. was supported by the Russian Found for Basic Research Grant 08-02-00768a, grants NS-5525.2010.2, MK-4317.2009.2, by the Federal Agency for Science and Innovation under state contract 02.740.11.0244, by the Federal Agency for Education under state

contracts P520 and P2598, by the Russian Science Support Foundation. Numerical part 
of the work was performed on the Computational cluster of the Theoretical Division of INR RAS.

\section{References}

[1] D.N. Spergel et al. [WMAP Collab.], "Wilkinson Microwave Anisotropy Probe (WMAP) three year results: implications for cosmology," Astrophys.J.Suppl. 170 (2007) 377 [arXiv:astro-ph/0603449].

[2] J.A. Tyson et al., "Detailed mass map of CL0024+1654 from strong lensing," Astrophys. J. 498 (1998) L107 (1998) [arXiv:astro-ph/9801193]; H. Dahle, "A compilation of weak gravitational lensing studies of clusters of galaxies," [arXiv:astro-ph/0701598].

[3] A. Borriello and P.Salucci et al., "The dark matter distribution in disc galaxies," Mon. Not. Roy. Astron. Soc. 323 (2001) 285; M. Persic et al., "The Universal rotation curve of spiral galaxies: 1. The Dark matter connection," Mon. Not. Roy. Astron. Soc. 281 (1996) 27.

[4] F. Zwicky, "Die Rotverschiebung von extragalaktischen Nebeln," Helv. Acta 6 (1933) 110.

[5] E. Komatsu et al. [WMAP Collab.], "Five-Year Wilkinson Microwave Anisotropy Probe (WMAP) Observations: Cosmological Interpretation," Astrophys.J.Suppl. 180, (2009) 330 [arXiv:astro-ph/0803.0547].

[6] S. Steigman and M. S. Turner, "Cosmological Constraints on the Properties of Weakly Interacting Massive Particles," Nucl. Phys. B253 (1985) 375.

[7] G. Jungman, M. Kamionkowski and K. Griest. "Supersymmetric dark matter," Phys. Rept. 267 (1996) 195 [arXiv:hep-ph/9506380].

[8] G. Bertone, D. Hooper, J. Silk, "Particle dark matter: Evidence, candidates and constraints," Phys. Rept. 405 (2005) 279 [arXiv:hep-ph/0404175].

[9] R. Bernabei et al. [DAMA Collab.], "First results from DAMA/LIBRA and the combined results with DAMA/NaI," Eur. Phys. J. C 56 (2008) 333 [arXiv:astro-ph/0804.2741].

[10] Z. Ahmed et al. [CDMS Collab.], "Search for Weakly Interacting Massive Particles with the First Five-Tower Data from the Cryogenic Dark Matter Search at the Soudan Underground Laboratory," Phys. Rev. Lett.102 (2009) 011301; Z. Ahmed et al. [CDMS Collab.], "Results from the Final Exposure of the CDMS II Experiment," [arXiv:astro-ph/0912.3592]. 
[11] G. Dobler and D. P. Finkbeiner, "Extended Anomalous Foreground Emission in the WMAP 3-Year Data," Astrophys. J. 680 (2008) 1222 [arXiv:astro-ph/0712.1038].

[12] W. de Boer et al., "The supersymmetric interpretation of the EGRET excess of diffuse Galactic gamma rays," Phys. Lett. 636 (2006) 13 [arXiv:astro-ph/0511154].

[13] W.B. Atwood et al. [Fermi/LAT Collab.], "The Large Area Telescope on the Fermi Gamma-ray Space Telescope Mission," Ap. J. 697 No 2 (2009) 1071; V. Vitale et al. [Fermi/LAT Collab.], "Indirect Search for Dark Matter from the center of the Milky Way with the Fermi-Large Area Telescope," Proc. of 2009 Fermi Symposium, Washington, D.C., (2009) [arXiv:astro-ph/0912.3828].

[14] P. Jean et al. [SPI/INTEGRAL Collab.], "Early SPI/INTEGRAL measurements of galactic $511 \mathrm{keV}$ line emission from positron annihilation," Astron. Astrophys. 407 (2003) L55 [arXiv:astro-ph/0309484]; N. Prantzos, "On the intensity and spatial morphology of the $511 \mathrm{keV}$ emission in the Milky Way," Astron. Astrophys. 449 (2006) 869 [arXiv:astro-ph/0511190].

[15] O. Adriani et al. [PAMELA Collab.], "Observation of an anomalous positron abundance in the cosmic radiation," Nature 458 (2009) 607 [arXiv:astro-ph/0810.4995].

[16] J. Chang et al. [ATIC Collab.], "An excess of cosmic ray electrons at energies of 300-800 GeV," Nature 456 (2008) 362.

[17] A. Bottino, F. Donato, N. Fornengo and S. Scopel, "Interpreting the recent results on direct search for dark matter particles in terms of relic neutralino," Phys. Rev. D 78 (2008) 083520 [arXiv:0806.4099 [hep-ph]].

[18] V. Niro, A. Bottino, N. Fornengo, S. Scopel, "Investigating light neutralinos at neutrino telescopes," Phys. Rev. D80 (2009) 095019 [arXiv:hep-ph/09092348].

[19] http://www.th.u-psud.fr/NMHDECAY/nmssmtools.html

[20] U. Ellwanger and C. Hugonie, "NMHDECAY 2.0: An Updated program for sparticle masses, Higgs masses, couplings and decay widths in the NMSSM," Comput. Phys. Commun. 175 (2006) 290 [arXiv:hep-ph/0508022].

[21] U. Ellwanger and C. Hugonie, "NMSPEC: A Fortran code for the sparticle and Higgs masses in the NMSSM with GUT scale boundary conditions," Comput. Phys. Commun. 177 (2007) 399 [arXiv:hep-ph/0612134].

[22] A. Djouadi et al., "Benchmark scenarios for the NMSSM," JHEP 0807 (2008) 002 [arXiv:0801.4321 [hep-ph]]. 
[23] G. Wikstrom and J. Edsjo, "Limits on the WIMP-nucleon scattering cross-section from neutrino telescopes," JCAP 0904 (2009) 009 [arXiv:0903.2986 [astro-ph.CO]].

[24] C. Hugonie, G. Belanger and A. Pukhov, "Dark Matter in the Constrained NMSSM," JCAP 0711 (2007) 009 [arXiv:0707.0628 [hep-ph]].

[25] G. Belanger, C. Hugonie and A. Pukhov, "Precision measurements, dark matter direct detection and LHC Higgs searches in a constrained NMSSM," JCAP 0901 (2009) 023 [arXiv:0811.3224 [hep-ph]].

[26] F. Ferrer, L. M. Krauss and S. Profumo, "Indirect detection of light neutralino dark matter in the NMSSM," Phys. Rev. D 74 (2006) 115007 [arXiv:hep-ph/0609257].

[27] M. Asplund, N. Grevesse, A. J. Sauval and P. Scott, "The chemical composition of the Sun," Ann. Rev. Astron. Astrophys. 47 (2009) 481 [arXiv:0909.0948 [astro-ph.SR]].

[28] G. Belanger, F. Boudjema, A. Pukhov and A. Semenov, "Dark matter direct detection rate in a generic model with micrOMEGAs2.1," Comput. Phys. Commun. 180 (2009) 747 [arXiv:0803.2360 [hep-ph]].

[29] M. M. Pavan, I. I. Strakovsky, R. L. Workman, R. A. Arndt, "The pion-nucleon Sigma term is definitely large: results from a G.W.U. analysis of pion nucleon scattering data," PiN Newslett. 16 (2002) 110-115 [arXiv:hep-ph/0111066v1]

[30] A. Gould, "Resonant Enhancements In Wimp Capture By The Earth," Astrophys. J. 321 (1987) 571.

[31] S. Sivertsson and J. Edsjo, "Accurate calculations of the WIMP halo around the Sun and prospects for its gamma-ray detection," Phys. Rev. D81 (2010) 063502 [arXiv:astro-ph.SR/0910.0017].

[32] K. Griest and D. Seckel, "Cosmic Asymmetry, Neutrinos and the Sun," Nucl. Phys. B 283 (1987) 681 [Erratum-ibid. B 296 (1988) 1034].

[33] A. Gould, "Cosmological density of WIMPs from solar and terrestrial annihilations," Astrophys. J. 388 (1992) 338.

[34] J. R. Ellis, K. A. Olive and C. Savage, "Hadronic Uncertainties in the Elastic Scattering of Supersymmetric Dark Matter,” Phys. Rev. D 77 (2008) 065026 [arXiv:0801.3656 [hep-ph]].

[35] J. Ellis, K. A. Olive, C. Savage and V. C. Spanos, "Neutrino Fluxes from CMSSM LSP Annihilations in the Sun," [arXiv:0912.3137 [hep-ph]]. 
[36] F. S. Ling, E. Nezri, E. Athanassoula and R. Teyssier, "Dark Matter Direct Detection Signals inferred from a Cosmological N-body Simulation with Baryons," JCAP 1002 (2010) 012 [arXiv:0909.2028 [astro-ph.GA]].

[37] M.M.Boliev et al., "Results with the Baksan neutrino telescope," Proc. of the First Workshop on Exotic Physics with Neutrino Telescope, Uppsala, Sweden, 19 (2006) [arXiv:astro-ph/0701333].

[38] J. Braun and D. Hubert for the IceCube Collaboration, "Searches for WIMP Dark Matter from the Sun with AMANDA," arXiv:0906.1615 [astro-ph.HE].

[39] M.M.Boliev et al., "Search for supersymmetric dark matter with Baksan Underground Telescope," Nucl. Phys., 48 (1996) 83.

[40] M.M.Boliev et al., "BAKSAN NEUTRALINO SEARCH," Dark Matter in Astro- and Particle Physics, edited by H.V.Klapdor-Kleingrothaus and Y.Ramachers (Singapore: World Sci.), (1997), 711; see also O.V.Suvorova, "Status and Perspectives of Indirect Search for Dark Matter," [arXiv:hep-ph/9911415].

[41] S. Desai et al., "Search for Dark Matter WIMPs using Upward Through-going Muons in Super-Kamiokande," Phys. Rev. D70 (2004) 083523.

[42] R. Abbasi et al. [Ice Cube Collab.], "Limits on a muon flux from neutralino annihilations in the Sun with the IceCube 22-string detector,"Phys.Rev.Lett.102 (2009) 201302, see also http://www.icecube.wisc.edu/news/.

[43] J. Angle et al. [XENON Collaboration], "First Results from the XENON10 Dark Matter Experiment at the Gran Sasso National Laboratory," Phys. Rev. Lett. 100 (2008) 021303 [arXiv:0706.0039 [astro-ph]].

[44] V. N. Lebedenko et al., "Result from the First Science Run of the ZEPLIN-III Dark Matter Search Experiment," Phys. Rev. D 80 (2009) 052010 [arXiv:0812.1150 [astro-ph]].

[45] S. Archambault et al., "Dark Matter Spin-Dependent Limits for WIMP Interactions on 19-F by PICASSO," Phys. Lett. B 682 (2009) 185 [arXiv:0907.0307 [hep-ex]].

[46] A. Avrorin et al., "Search for Neutrinos from Dark Matter Annihilation in the Sun with the Baikal Neutrino Experiment," Proc. of the 31st ICRC, Lodz, Poland (2009) [arXiv:0909.5589v1 [astro-ph.HE]]

[47] G. Lim for the ANTARES Collaboration, "First results on the search for dark matter in the Sun with the ANTARES neutrino telescope," Proc. of the 31st ICRC, Lodz, Poland (2009) [arXiv:0905.2316v3 [astro-ph.CO]] 
[48] http://dmtools.brown.edu

[49] M. Cirelli, N. Fornengo, T. Montaruli, I. Sokalski, A. Strumia and F. Vissani, "Spectra of neutrinos from dark matter annihilations," Nucl. Phys. B 727 (2005) 99 [Erratum-ibid. B 790 (2008) 338] [arXiv:hep-ph/0506298]; M. Blennow, J. Edsjo and T. Ohlsson, "Neutrinos from WIMP Annihilations Using a Full Three-Flavor Monte Carlo," JCAP 0801 (2008) 021 [arXiv:0709.3898 [hep-ph]]. 

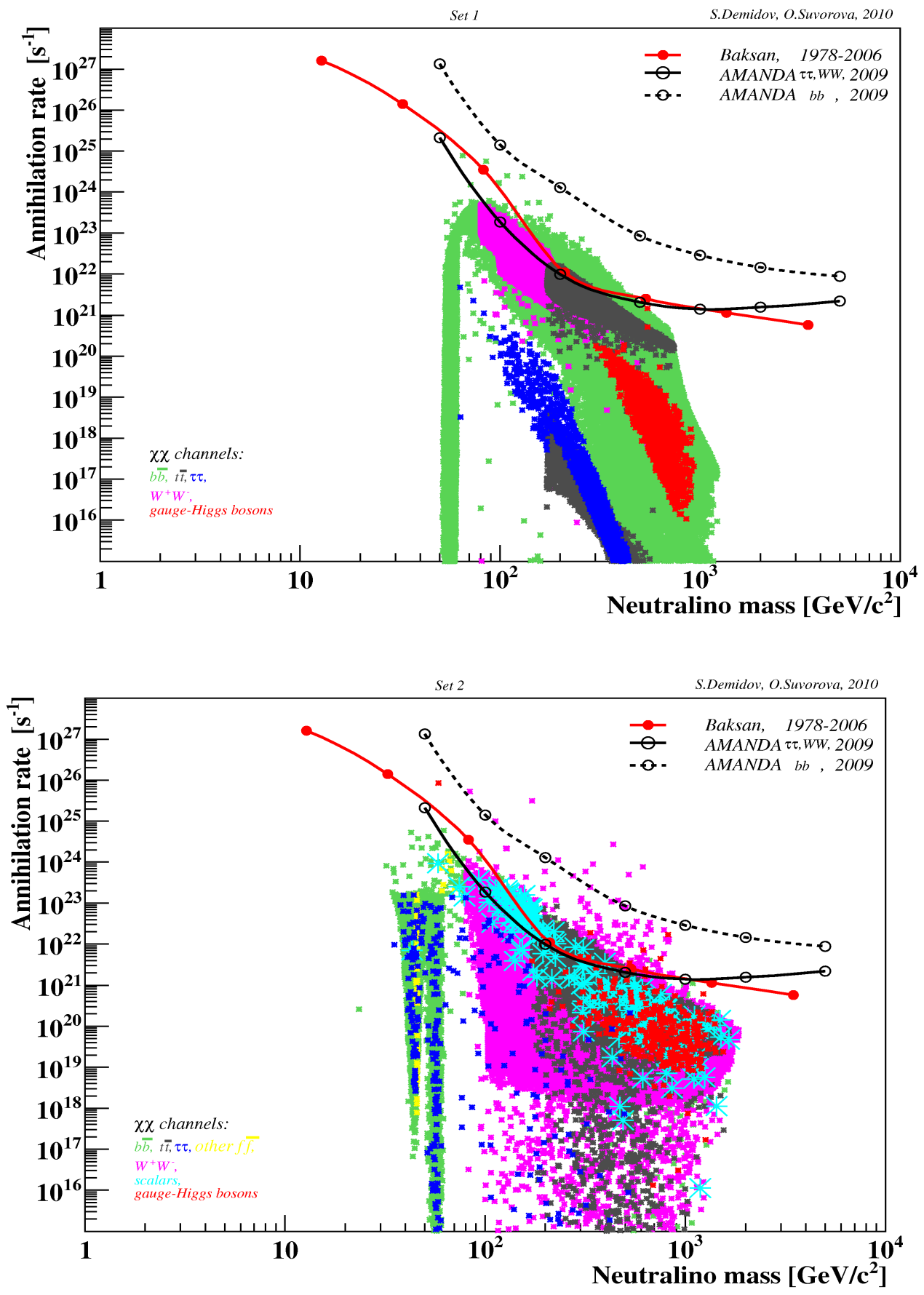

Figure 1: Annihilation rates vs neutralino mass for Set 1 (up) and Set 2 (bottom) of parameters. Here all points are in the interval $0<\Omega_{\chi}<0.3$. We show the dominant annihilation channel $(\mathrm{Br}>0.5)$ by different colours. 

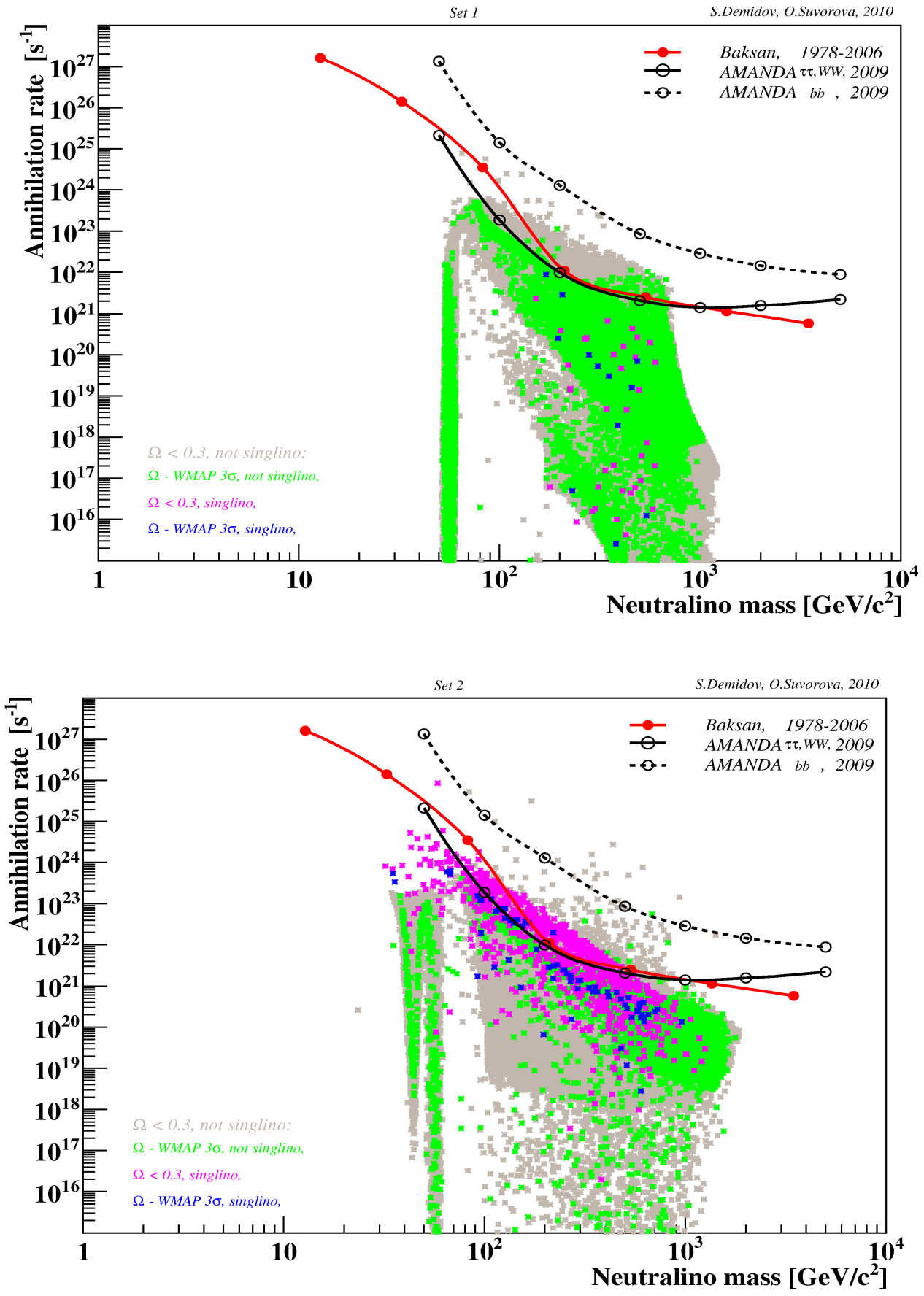

Figure 2: Annihilation rates vs neutralino mass for Set 1 (up) and Set 2 (bottom) of parameters. 

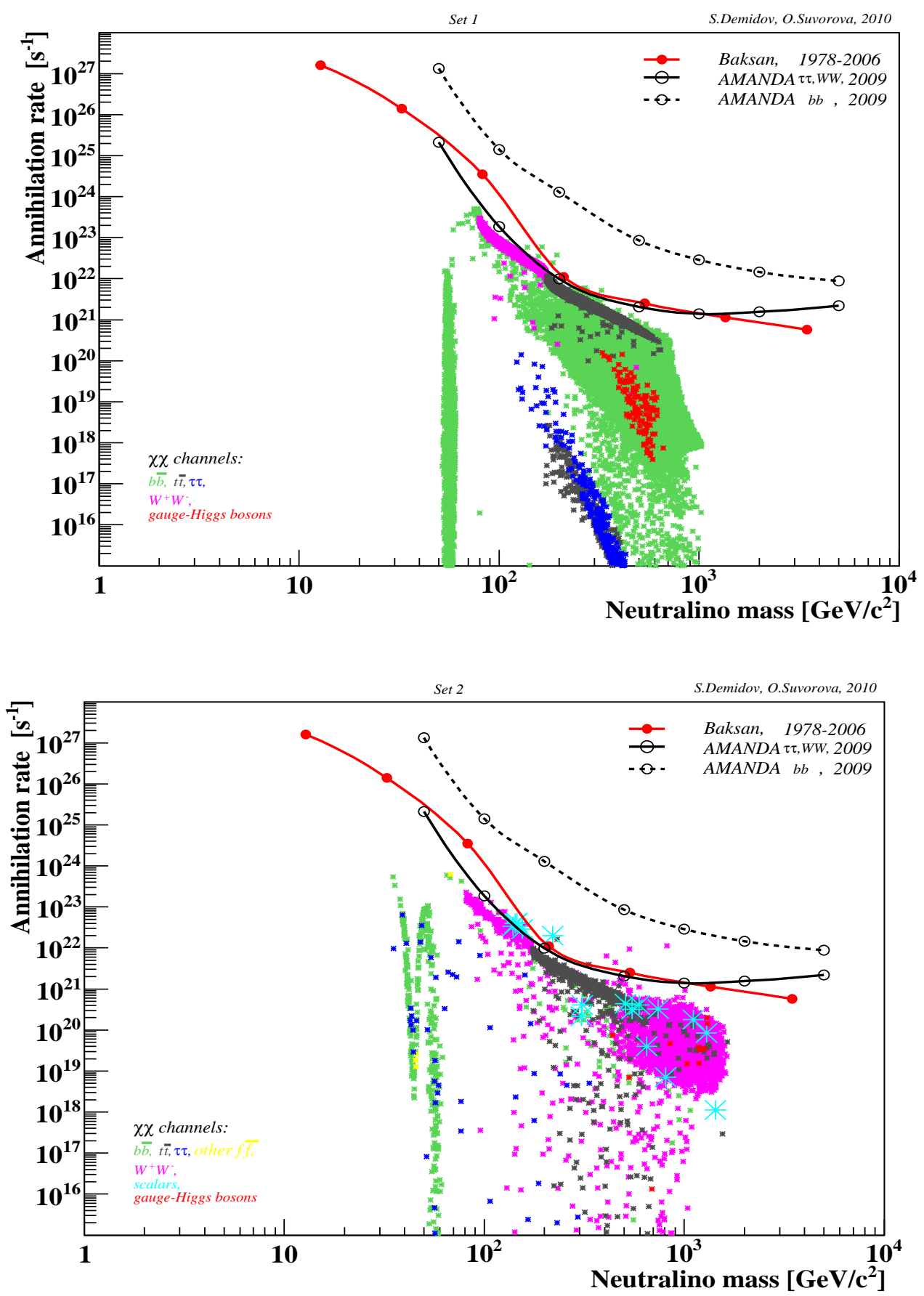

Figure 3: Annihilation rates vs neutralino mass for Set 1 (up) and Set 2 (bottom) of parameters. Here all points are in $3 \sigma$ WMAP interval: $0.0913<\Omega_{D M}<0.1285$. We show the dominant annihilation channel $(\mathrm{Br}>0.5)$ by different colours. 

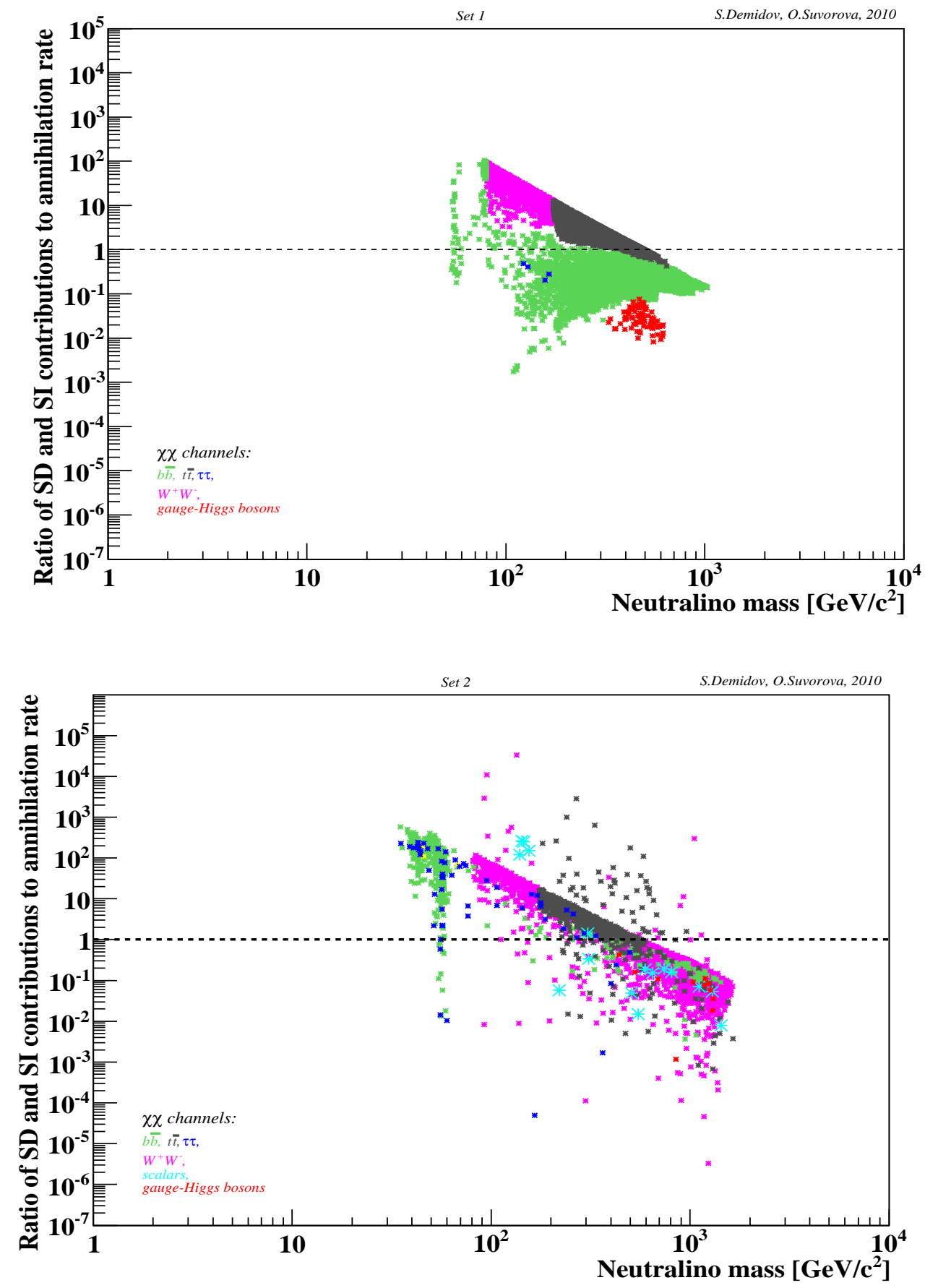

Figure 4: Comparison of relative contribution of spin-dependent and spin-independent interactions to annihilation rate. All conventions are the same as in Fig. 3 . 

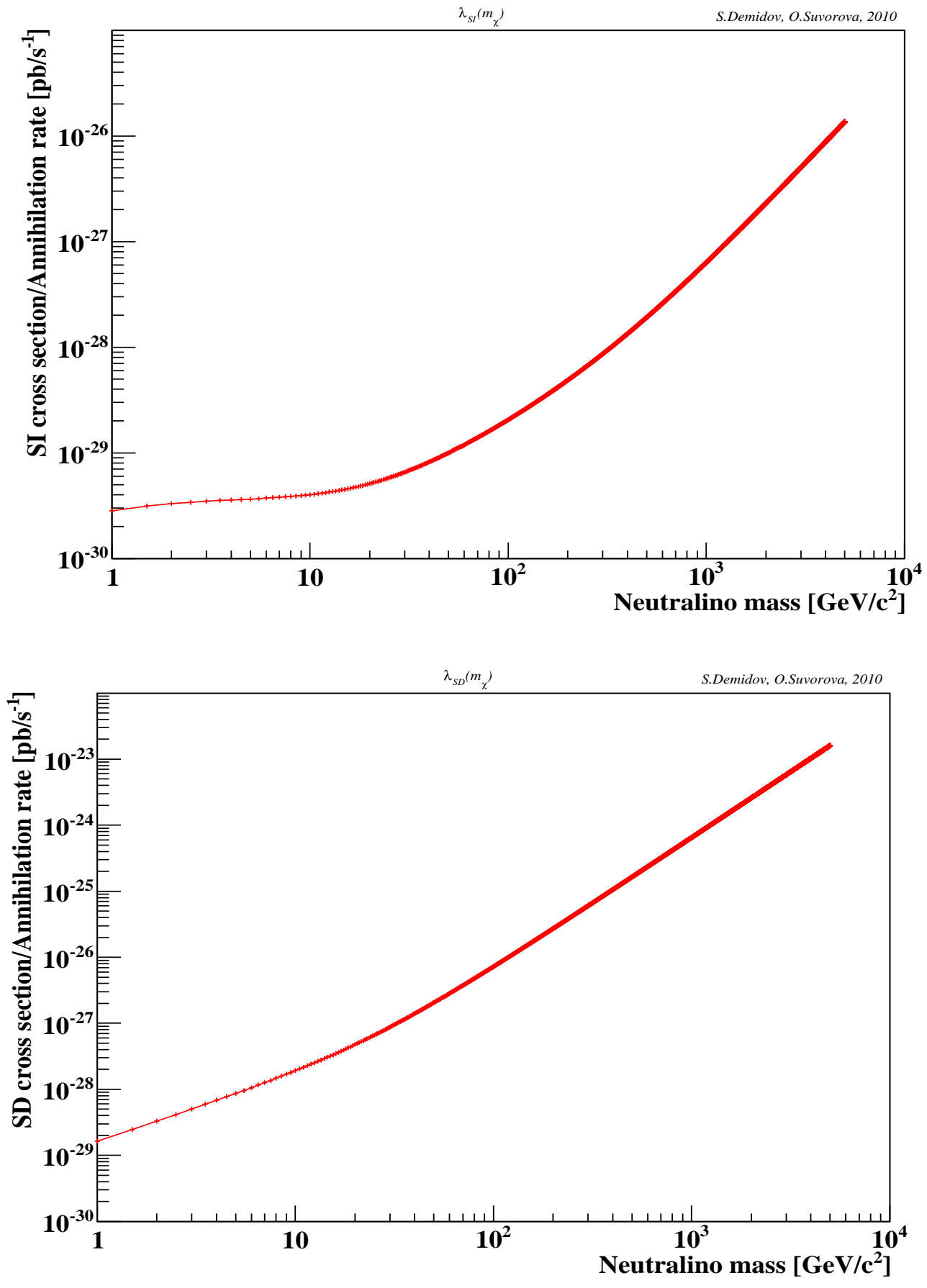

Figure 5: Coefficients $\lambda_{S I}\left(m_{\chi}\right)$ (up) and $\lambda_{S D}\left(m_{\chi}\right)$ (bottom). 

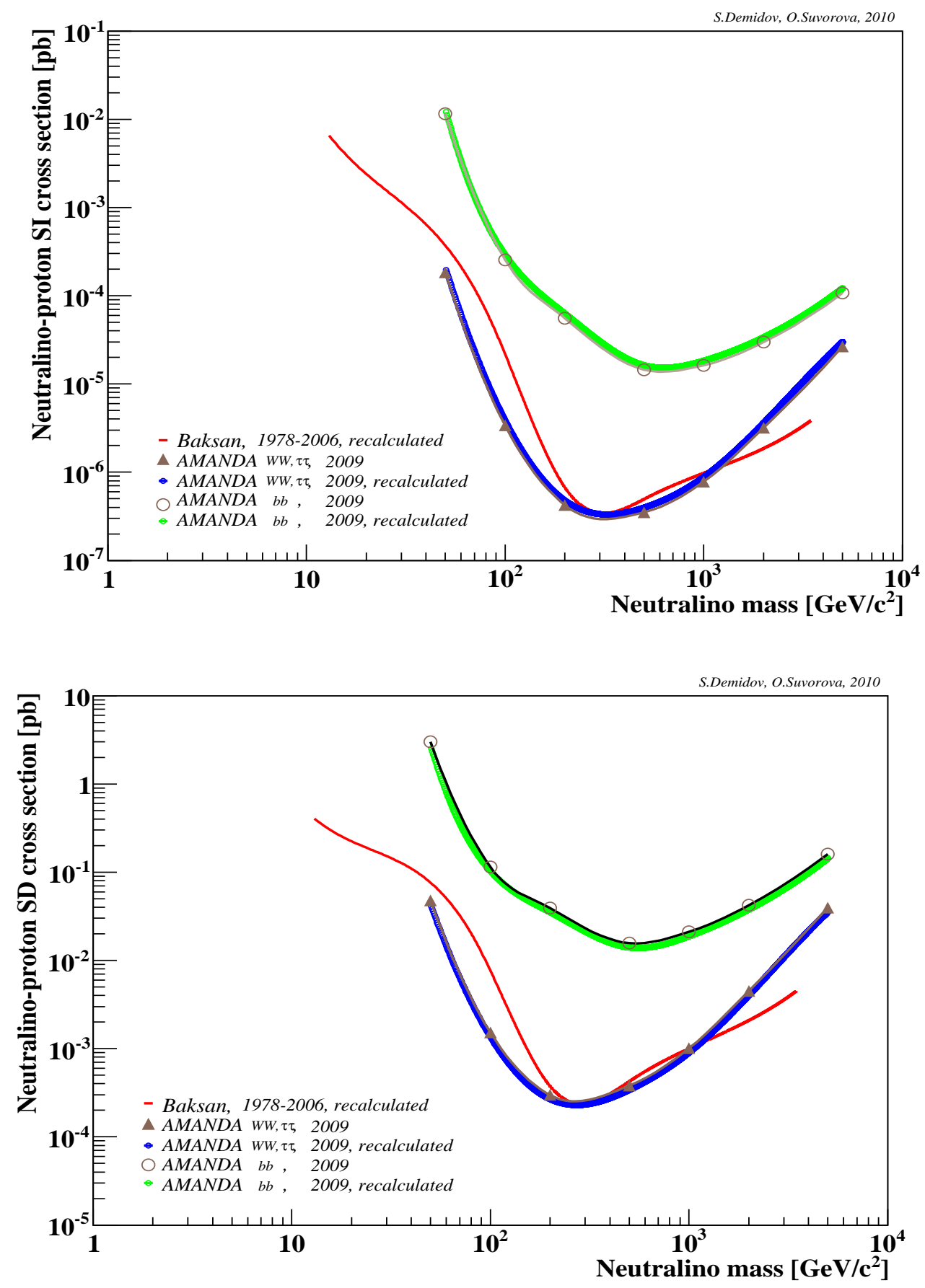

Figure 6: The upper limits at $90 \%$ c.l. on neutralino-proton spin-independent (up) and spin-dependent (bottom) cross sections for the Baksan underground scintillator telescope recalculated from their $90 \%$ c.l. limits on annihilation rates [37] (red lines). Also shown are the AMANDA upper limits at $90 \%$ c.l. on SI and SD cross sections presented in 38] (triangles) and our recalculated values from the AMANDA upper limits on annihilation rates (blue lines). 

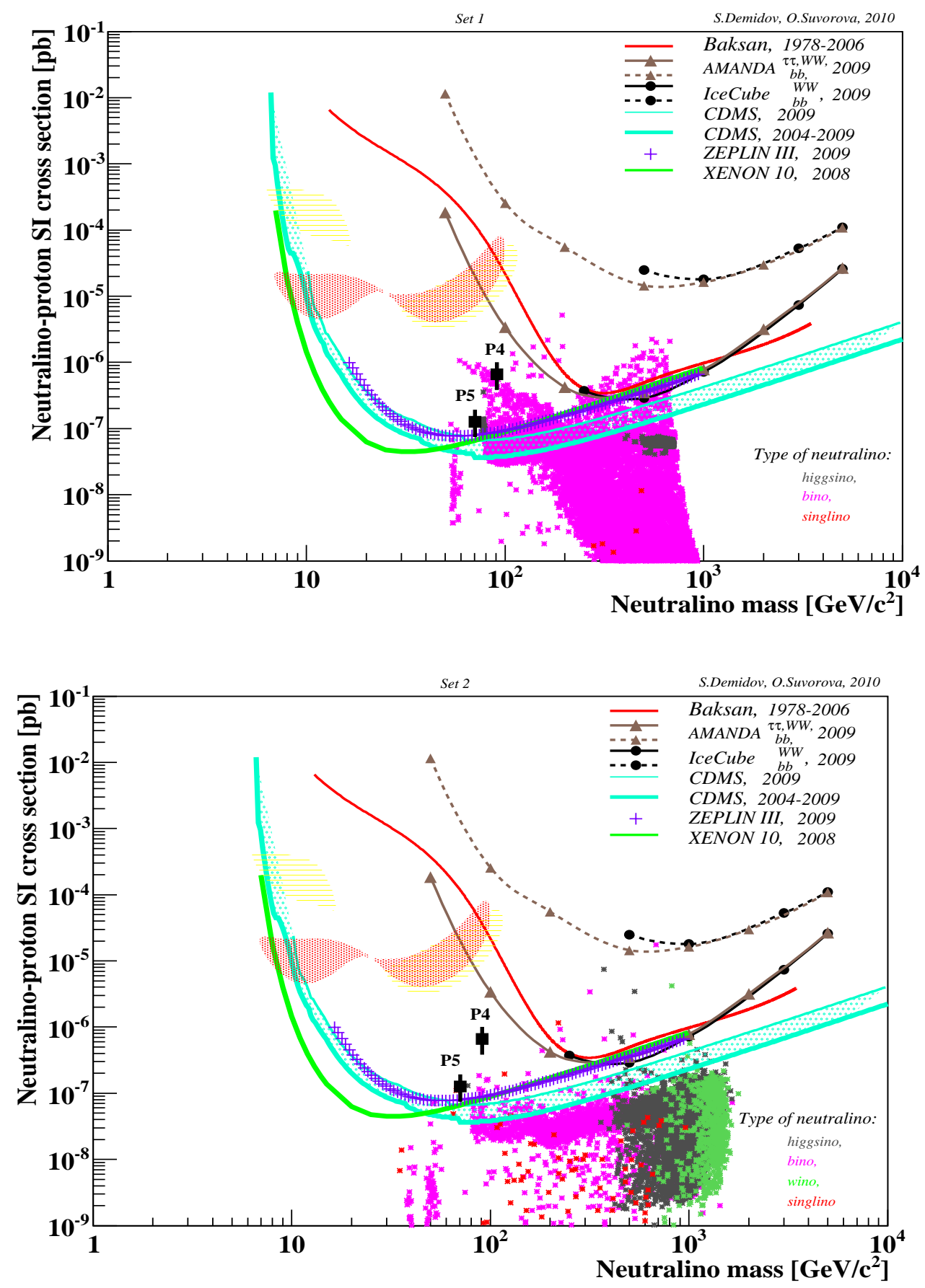

Figure 7: Neutralino-proton spin-independent cross section vs neutralino mass for Set 1 (up) and Set 2 (bottom) of parameters and experimental limits at $90 \%$ c.l. Neutralino content is shown by different colours. 

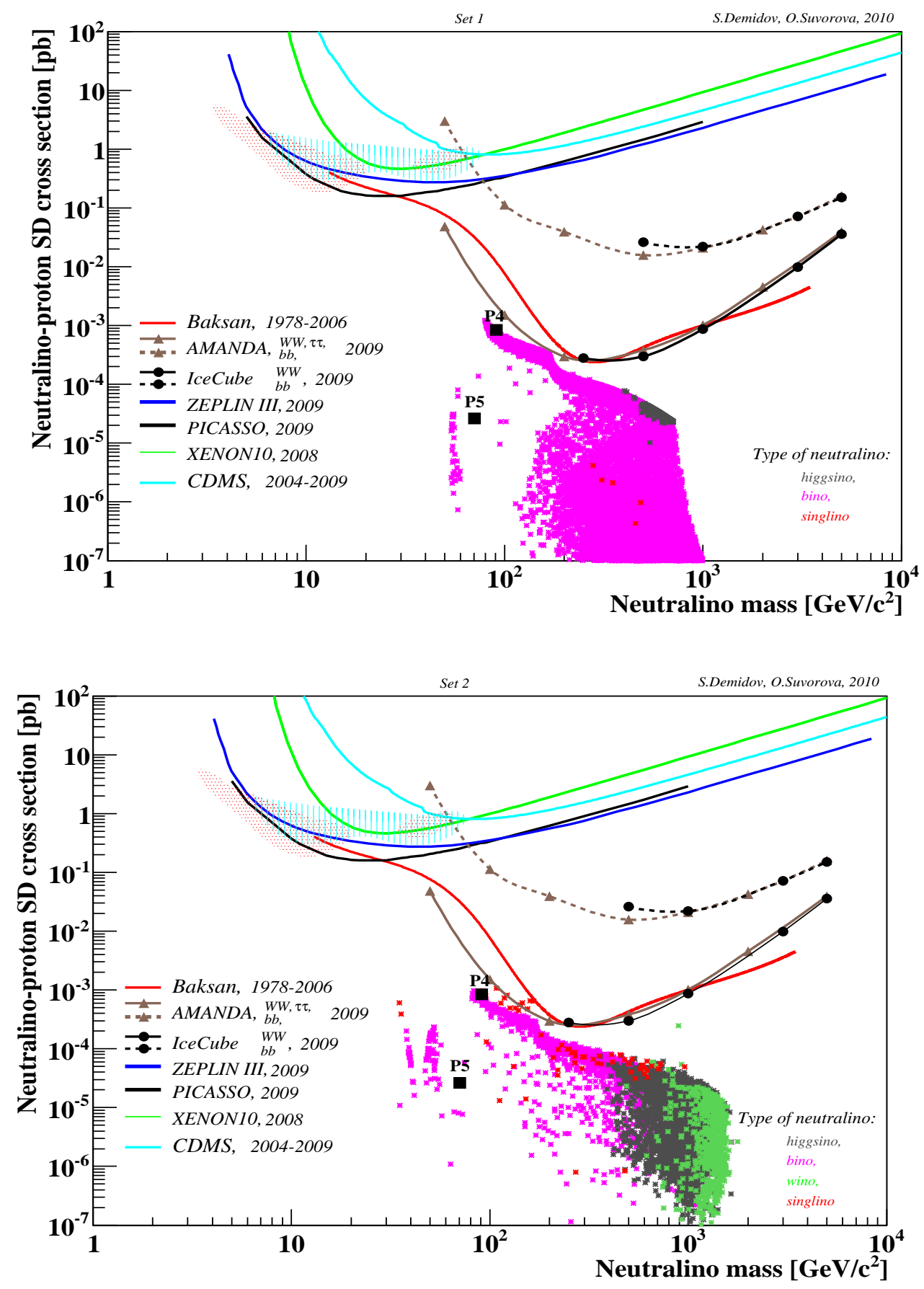

Figure 8: Neutralino-proton spin-dependent cross section vs neutralino mass for Set 1 (up) and Set 2 (bottom) of parameters and experimental limits. The notations are as in Fig.7. All points are constraint by SI cross section experimental limits at $90 \%$ c.l. 